\title{
Population-based studies in systemic lupus erythematosus: immune thrombocytopenic purpura or 'blood- dominant' lupus?
}

\author{
Antonis Fanouriakis $\odot,{ }^{1}$ George Bertsias, ${ }^{2}$ Dimitrios T Boumpas $\odot$
}

Thanks to their large sample size, thus reducing the risk of selection and participation bias, population-based studies can provide high-quality data on the prevalence and incidence, natural history and treatment, correlates and associations of a disease, and healthcare utilisation. ${ }^{1}$ Several nationwide research databases exist in the world, one of the oldest in Sweden dating back to 1955. The Taiwan National Health Insurance Research Database (NHIRD) is one of the largest nationwide population databases, covering approximately 23 million residents in Taiwan and data of more than $99 \%$ of the population. Using this unique database, investigators have asked important questions regarding the heredity and coaggregation of autoimmune diseases, such as systemic lupus erythematosus (SLE), Sjögren's syndrome and myasthenia gravis. ${ }^{2-4}$

Immune thrombocytopenic purpura (ITP), formerly known as idiopathic thrombocytopenic purpura, is an immunemediated acquired disease of adults and children characterised by a transient or persistent decrease of platelet counts and, depending on the degree of thrombocytopenia, increased risk of bleeding. ${ }^{5}$ In ITP, an abnormal $\mathrm{T}$ cell response, supported by splenic $\mathrm{T}$ follicular helper cells, stimulates the proliferation and differentiation of autoreactive $\mathrm{B}$ cells producing antiplatelet autoantibodies that facilitate platelet phagocytosis by macrophages, predominantly in the spleen. Macrophages also contribute to the perpetuation of the autoimmune response in ITP, functioning as the principal antigen-presenting cells. Inappropriate bone marrow production

'Department of Rheumatology, "Asklepieion" General Hospital, Athens, Greece

${ }^{2}$ Rheumatology, Clinical Immunology and Allergy, University Hospital of Heraklion, Heraklion, Greece ${ }^{3}$ Rheumatology and Clinical Immunology Unit, 4th Department of Internal Medicine, University Hospital "Attikon", Athens, Greece

Correspondence to Dr Dimitrios T Boumpas, Rheumatology and Clinical Immunology Unit, 4th Department of Internal Medicine, University Hospital "Attikon", Chaidari 124 62, Greece; boumpasd@uoc.gr due to an immune response against megakaryocytes may also exacerbate thrombocytopenia, while the level of circulating thrombopoietin, the main growth factor of megakaryocytes, is low. ${ }^{6}$ Genomewide expression analyses have shown dysregulation of genes involved in major immune response pathways in ITP, such as $\mathrm{T}$ helper cell activation and differentiation, autoantibody response and complement activation both in peripheral and bone marrow-derived T-cells. ${ }^{78}$ Autoantibodies against platelet antigens represent the diagnostic hallmark of ITP, but are detectable in only $50 \%$ of patients. Using mass spectroscopy-based antibodymediated identification of autoantigens for platelet antigens, novel autoantigens have been identified from patients who are autoantibody negative. Most antigens are of intracellular origin, with significant association with the actin cytoskeleton and regulation of programmed cell death. ${ }^{9}$ Taken together, the aetiopathogenetic mechanisms operant in ITP, a prototypic organ-specific autoimmune disease, are reminiscent of those described in the prototypic systemic autoimmune disease, namely SLE. Abberancies in immune cells in SLE can also be traced back to the haematopoietic progenitor stem cells in the bone marrow. ${ }^{10}$

Thrombocytopenia is a common clinical manifestation of SLE and one of the haematological classification criteria, with an overall prevalence of approximately $20 \%$. In a recent community-based SLE cohort of average disease duration less than 3 years, thrombocytopenia was present in $15.2 \%$ of patients, being severe ( $\leq 20000$ platelets/ $\mu \mathrm{L})$ in $4.6 \% .{ }^{11}$ In our tertiary referral centre cohort (the 'Attikon' cohort), thrombocytopenia was present at diagnosis in $12 \%$ and cumulatively in $16 \% .{ }^{12}$ Notably, in an older study, $\sim 12 \%$ of cases had been initially diagnosed as ITP. ${ }^{13}$

In Annals of the Rheumatic Diseases, Zhu et al used data from the Taiwan NHIRD to estimate the risk for subsequent (incident) SLE among patients with an initial diagnosis of ITP. $^{14}$ The authors selected 1070 patients hospitalised during 1997-2013 with ITP, of whom 668 were finally included in the study. Next, they matched patients with ITP at a ratio of $1: 20$ to 14.460 controls by sex and age, using propensity score matching at a $1: 2$ ratio to minimise the potential confounding effects of age, gender and selected comorbidities on the incidence of SLE. After a follow-up of 16 years, patients with ITP had 26.8 times higher risk of new-onset SLE. The risk was higher for patients aged less than 45 years and lower for men. Not surprising, patients with Sjögren's syndrome or vasculitis had a significantly higher risk for SLE.

These findings have important clinical implications. First and foremost, patients with ITP-especially those who are antinuclear antibody (ANA) positive-should be closely monitored for SLE, especially within the first 2-5 years of diagnosis, and should probably be evaluated by a rheumatologist for the identification of non-haematological signs and symptoms suggestive of a connective tissue disease (figure 1). In our experience, non-rheumatologists may miss subtle signs of SLE, such as synovitis and inflammatory rashes, or they may fail to evaluate lupus-related signs that do not occur concurrently with thrombocytopenia. Testing for SLE serology and antiphospholipid antibodies, serum creatinine and urinalysis should be considered in all cases of ITP. Several studies have shown that thrombocytopenia is associated with worse SLE prognosis; ${ }^{15} 16$ hence, early recognition and management of SLE, especially renal disease, are essential. ${ }^{17}$ In children with chronic ITP (as opposed to acute ITP), routine check for SLE and evaluation by a paediatric rheumatologist may also be advisable.

Second, although in general terms, SLErelated thrombocytopenia and ITP are managed initially with identical drugs, recognising and diagnosing an underlying lupus may lead to reconsideration of thoughts for splenectomy (ie, postponing it for later) in patients with refractory disease and may lead to the use, in addition to rituximab, of SLE-directed therapies, like pulse cyclophosphamide (figure 1). ${ }^{17} 18$ Moreover, patients who are ANA positive or have additional but not enough features to allow a confident diagnosis or classification of SLE (incomplete SLE) may be considered for treatment with hydroxychloroquine. In this regard, a small study from France with 40 patients with ITP and ANA positivity and/ or lupus-like features who were refractory to initial therapy has shown efficacy in 


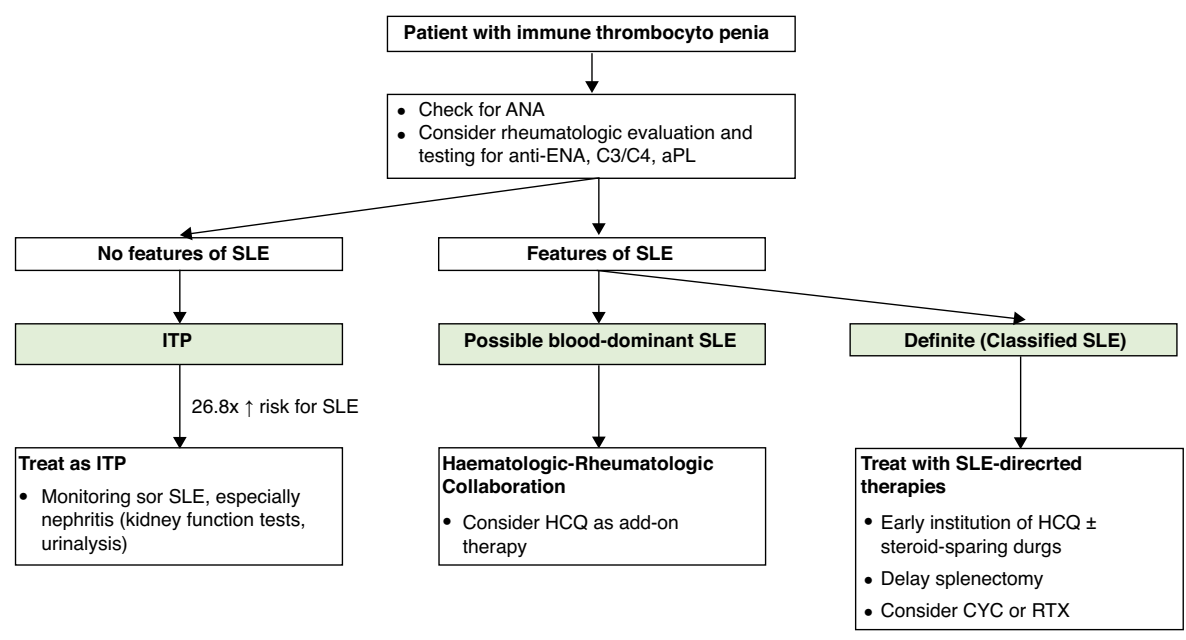

Figure 1 Approach to the patient who presents with immune thrombocytopenia. n.b. Thrombocytopenia $\left(<100000 / \mathrm{mm}^{3}\right)$ is present in $\sim 10 \%$ of patients with systemic lupus erythematosus (SLE) at diagnosis and in $~ 15-20 \%$ cumulatively over the course of the disease. ANA, antinuclear antibodies; anti-ENA, antibodies to extractable nuclear antigens; aPL, antiphospholipid antibodies; CYC, cyclophosphamide; HCQ, hydroxychloroquine; ITP, immune thrombocytopenic purpura; RTX, rituximab.

glucocorticoid sparing and prevention of flares. ${ }^{19}$

Finally, this study also poses the important question: is the concurrence of ITP and SLE related to a common genetic background shared by several autoimmune diseases,${ }^{20}$ or is it due to the fact that several cases of SLE represent incomplete forms of the disease or a 'blood-dominant' lupus. ${ }^{21}$ To this end, studies comparing molecular signatures of these two diseases may help to decipher this, as signatures specific for SLE such as type I interferon or plasmablasts, ${ }^{22}$ may not be present in pure ITP.

In conclusion, population-based studies offer the unique opportunity to provide high-quality evidence regarding, among others, the heredity and coaggregation of autoimmune diseases with apparent clinical implications.

\section{Handling editor Josef S Smolen \\ Twitter Dimitrios T Boumpas @none}

Contributors DTB drafted the manuscript. AF and GB edited the manuscript. All authors read and approved its final form.

Funding The authors have not declared a specific grant for this research from any funding agency in the public, commercial or not-for-profit sectors.

Competing interests None declared.

Patient and public involvement Patients and/ or the public were not involved in the design, or conduct, or reporting, or dissemination plans of this research.

Patient consent for publication Not required.

Provenance and peer review Commissioned; internally peer reviewed.
5 Cines DB, Bussel JB, Liebman HA, et al. The ITP syndrome: pathogenic and clinical diversity. Blood 2009;113:6511-21.

6 Audia S, Mahévas M, Samson M, et al. Pathogenesis of immune thrombocytopenia. Autoimmun Rev 2017;16:620-32.

7 Jernås M, Nookaew I, Wadenvik H, et al. Differential expression of T-cell genes in blood and bone marrow between ITP patients and controls. Thromb Haemost 2013;109:112-7.

8 Jernås $\mathrm{M}$, Nookaew I, Wadenvik $\mathrm{H}$, et al. Microrna regulate immunological pathways in T-cells in immune thrombocytopenia (ITP). Blood 2013;121:2095-8.

9 Bal G, Futschik ME, Hartl D, et al. Identification of novel biomarkers in chronic immune thrombocytopenia (ITP) by microarray-based serum protein profiling. $\mathrm{Br} J$ Haematol 2016;172:602-15.

10 Grigoriou M, Banos A, Filia A, et al. Transcriptome reprogramming and myeloid skewing in haematopoietic stem and progenitor cells in systemic lupus erythematosus. Ann Rheum Dis 2020:79:242-53.

11 Adamichou C, Nikolopoulos D, Genitsaridi I, et al. In an early SLE cohort the ACR-1997, SLICC-2012 and EULAR/ACR-2019 criteria classify non-overlapping groups of patients: use of all three criteria ensures optimal capture for clinical studies while their modification earlier classification and treatment. Ann Rheum Dis 2020;79:232-41.

12 Nikolopoulos D, Kostopoulou M, Pieta A, et al. Evolving phenotype of systemic lupus erythematosus in Caucasians: low incidence of lupus nephritis, high burden of neuropsychiatric disease and increased rates of late-onset lupus in the 'Attikon' cohort. Lupus 2020:39:096120332090893.

To cite Fanouriakis A, Bertsias G, Boumpas DT. Ann Rheum Dis 2020;79:683-684.

Received 28 March 2020

Revised 2 April 2020

Accepted 2 April 2020

Published Online First 20 April 2020

\section{(S) Linked}

- http://dx.doi.org/10.1136/annrheumdis-2020217013

Ann Rheum Dis 2020;79:683-684.

doi:10.1136/annrheumdis-2020-217356

\section{ORCID iDs}

Antonis Fanouriakis http://orcid.org/0000-0003-2696031X

Dimitrios T Boumpas http://orcid.org/0000-0002-98124671

\section{REFERENCES}

1 Hsing AW, loannidis JPA. Nationwide population science: lessons from the Taiwan National health insurance research database. JAMA Intern Med 2015:175:1527.

2 Kuo C-F, Grainge MJ, Valdes AM, et al. Familial aggregation of systemic lupus erythematosus and coaggregation of autoimmune diseases in affected families. JAMA Intern Med 2015;175:1518.

3 Kuo C-F, Grainge MJ, Valdes AM, et al. Familial risk of Sjögren's syndrome and co-aggregation of autoimmune diseases in affected families: a nationwide population study. Arthritis Rheumatol 2015;67:1904-12

4 Liu F-C, Kuo C-F, See L-C, et al. Familial aggregation of myasthenia gravis in affected families: a population-based study. Clin Epidemiol 2017:9:527-35. 